\title{
Familial Thoracic Aortic Aneurysm and Dissection Associated with Marfan-related Gene Mutations: Case Report of a Family with Two Gene Mutations
}

\author{
Tohru Yamawaki ${ }^{1}$, Kazuhiro Nagaoka ${ }^{1}$, Kunio Morishige ${ }^{1}$, Kenji Sadamatsu ${ }^{1}$, \\ Hideki Tashiro ${ }^{1}$, Hiroshi Yasunaga ${ }^{2}$, Hiroko Morisaki ${ }^{3}$ and Takayuki Morisaki ${ }^{3}$
}

\begin{abstract}
We report three cases of thoracic aortic aneurysm and dissection in a Japanese family. Marfan-related genes were analyzed; FBN1 and TGFBR2 gene mutations were observed in this family.
\end{abstract}

Key words: thoracic aortic aneurysm and dissection, Marfan's syndrome, Loeys-Dietz syndrome, FBN1, TGFBR2, TGF- $\beta$

(Inter Med 48: 555-558, 2009)

(DOI: 10.2169/internalmedicine.48.1833)

\section{Introduction}

Thoracic aortic aneurysm and dissection (TAAD) can be inherited in an autosomal dominant manner. Marfan's syndrome (MFS) is a genetic disorder which is the result of inherited TAAD. MFS is a connective tissue disorder with involvement of the cardiovascular, ocular, and skeletal systems, caused by mutations in the fibrillin-1 (FBN1) gene on chromosome $15 q(1,2)$. The clinical diagnosis of MFS is currently based on the Ghent nosology, however the phenotypes that do not fulfill the criteria exist, and currently are often termed "type-1 fibrillinopathy" $(3,4)$. Recently, mutations in the gene for transforming growth factor-beta (TGF$\beta$ ) receptor type 1 and 2 (TGFBR1/2) have been described in patients with MFS and Marfan-like connective tissue disorders, including Loeys-Dietz syndrome (LDS) $(5,6)$.

Here, we report familial TAAD cases in which we were able to analyze these gene mutations.

\section{Case Report}

<Case 1> A 37-year-old man (height, $190 \mathrm{~cm}$; weight, 72 $\mathrm{kg}$ ) was referred for dyspnea following momentary chest pain in February 2004. A chest X-ray showed pulmonary congestion and trans-thoracic echocardiography showed aortic valve regurgitation without ascending aortic dissection. Computed tomography (CT) demonstrated aortic dissection from the aortic arch to the bilateral common iliac arteries, not in the ascending aorta (Fig. 1A). However, transesophageal echocardiography revealed a circumferential intimal flap with valve-like motion in the dilated aortic root and severe aortic regurgitation (Fig. 1B). The Bentall procedure was urgently performed. The post-operative course was uncomplicated, and he has remained asymptomatic (7).

$<$ Case 2> A 41-year-old woman (height, $165 \mathrm{~cm}$; weight, $56 \mathrm{~kg}$ ) in the 36th week of pregnancy was referred for new onset severe back pain in August 2007. Her blood pressure, cardiac enzymes, blood gases, electrocardiogram were normal. Echocardiography showed no abnormality in the heart and ascending aorta, but a dissection in the abdominal aorta. Magnetic resonance imaging revealed acute type B aortic dissection from distal aortic arch to abdominal aorta (Fig. 1C). She had a large false lumen, however, complications such as mal-perfusion of major branches or bleeding were not detected on CT using contrast medium (Fig. 1D, E). Therefore, she was treated with a beta-blocker, and underwent elective cesarean section in the 38th week of pregnancy. The post-delivery course was uneventful for the mother and baby.

${ }^{1}$ Division of Cardiovascular Medicine, St. Mary's Hospital, Kurume, ${ }^{2}$ Division of Cardiovascular Surgery, St. Mary's Hospital, Kurume and ${ }^{3}$ Department of Bioscience, National Cardiovascular Center, Suita

Received for publication November 1, 2008; Accepted for publication December 15, 2008

Correspondence to Dr. Tohru Yamawaki, tohru@ orange.ocn.ne.jp 

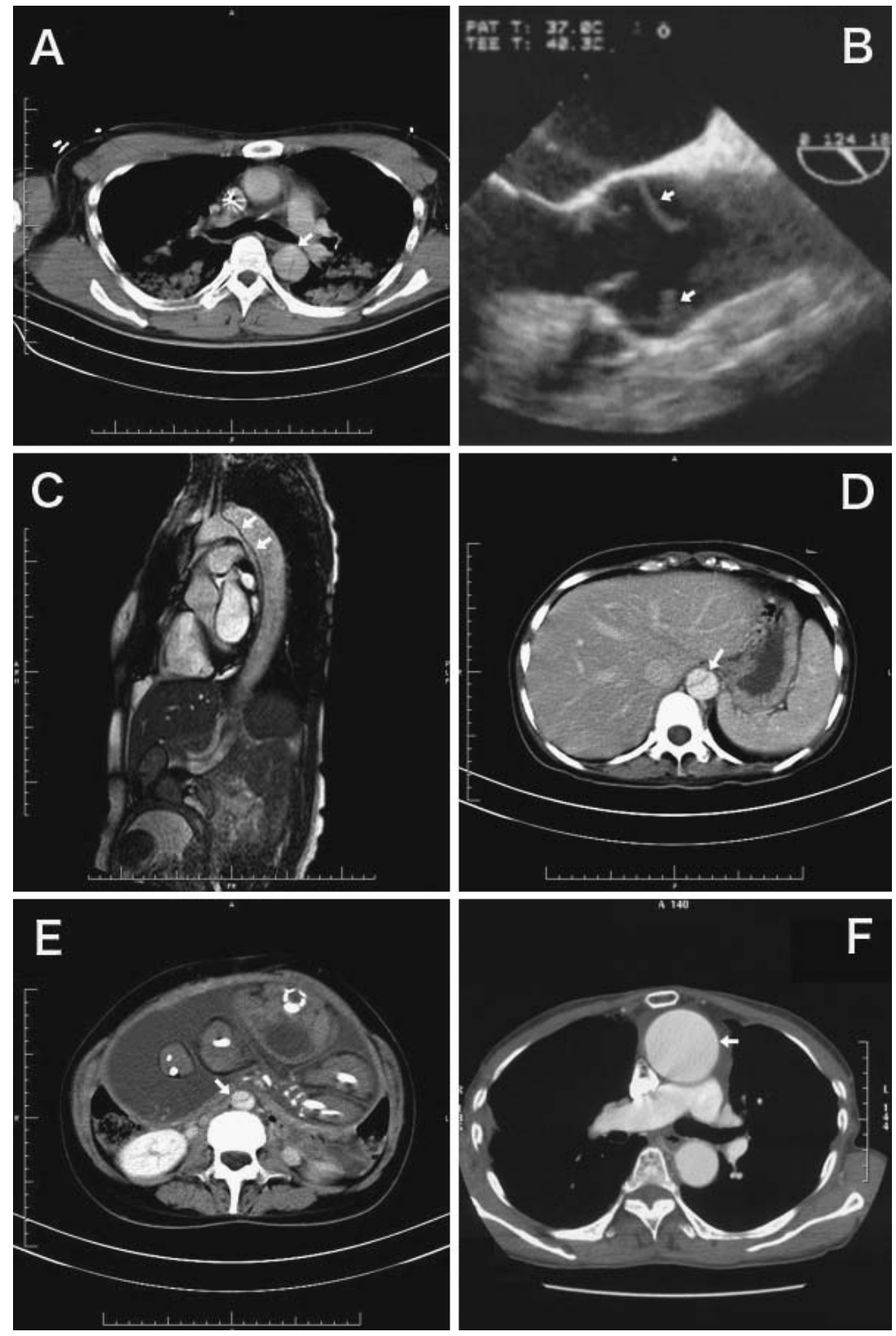

Figure 1. A: Contrast CT showed aortic dissection of the descending aorta (arrow) in Case 1. B.: Trans-esophageal echocardiography showed a circumferential intimal flap (arrows) with valve-like motion in the dilated aortic root in Case 1. C : Magnetic resonance imaging showed acute type B aortic dissection from distal aortic arch to abdominal aorta (arrows) in Case 2. D, E: Contrast CT showed aortic dissection of abdominal aorta (arrows) in Case 2. In addition, it was shown that her baby was in the abdomen. $\mathrm{F}$ : Contrast $\mathrm{CT}$ showed a thoracic aortic aneurysm that was $47 \mathrm{~mm}$ in diameter (arrows) in Case 3.

<Case 3> A 69-year-old woman (height, $166 \mathrm{~cm}$; weight, $42 \mathrm{~kg}$ ) was admitted for investigation of an abnormal cardiac silhouette on chest $\mathrm{x}$-ray film in 2006. She had no chest symptoms. CT revealed a thoracic aortic aneurysm that was $47 \mathrm{~mm}$ in diameter (Fig. 1F). She had received a beta-blocker, however, suddenly died due to rupture of the aneurysm in November 2007.

Case 1 and Case 2 were cousins, and Case 3 was the mother of Case 2. They were normotensive, comparatively tall and showed a positive wrist sign (thumb and fifth finger overlap when encircling the wrist), however, they did not have scoliosis or ocular disorders which are major criteria of MFS. The ratio of arm span/height was over 1.05 only in Case 2. We examined Case 2 in detail, and admitted a characteristic face in MFS with dental crowding, a flatfoot, a wide uvula and translucent skin. In additional, sudden death, aortic dissections and scoliosis occurred in the brothers of Case 3 (Fig. 2). We considered them as hereditary TAAD related to MFS though they did not completely fill the Ghent criteria, and analyzed the gene mutations associated with MFS and related phenotypes. All 3 cases and several of their children had the FBN1 gene mutation $7,115 \mathrm{G}=>\mathrm{T}$ in 


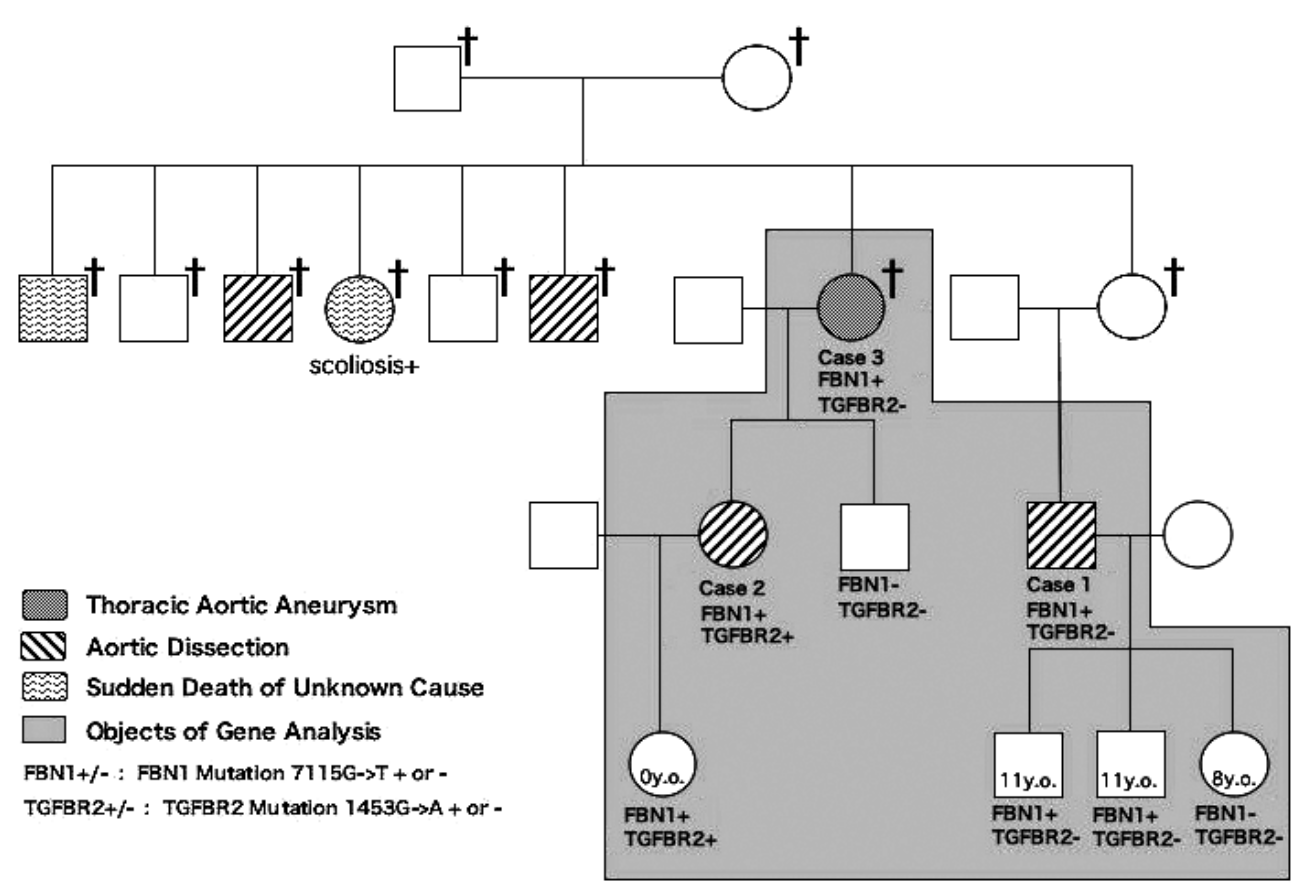

Figure 2. Family tree of the thoracic aortic aneurysm and dissection family.

exon 57, and glycine to valine replacement at amino acid 2,372 (p. Gly2372Val). Additionally, we found the TGFBR2 gene mutation $1,453 \mathrm{G}=>\mathrm{A}$ in exon 6 (p. Glu485Lys) in Case 2 and her child. These mutations were not detected in the younger brother of Case 2 who had no symptoms (Fig. 2).

\section{Discussion}

We encountered hereditary TAAD cases. They had a FBN1 gene mutation, and, in addition, one of them had a TGFBR2 gene mutation. These mutations resulted in missense coding changes.

FBN1 gene mutations have been shown to cause MFS as well as a series of other related disorders called "type-1 fibrillinopathy". More than 600 mutations on the FBN1 gene have been identified [Universal Mutation Database (UMD), www.umd.necker.fr], and the majority of these mutations are unique to the affected families, the mutation $7,115 \mathrm{G}=>\mathrm{T}$ had not been previously reported. Every patient with a FBN1 mutation may have a risk for developing cardiovascular, skeletal, and ocular complications. This novel mutation on FBN1 may be a cause of familial TAAD (because it was not inherited in the younger brother of Case 2 who had no abnormal manifestation in this family) (4).

In this report, Case 2 and her child had a TGFBR2 gene mutation in addition to a FBN1 gene mutation. TGFBR2 gene mutations have recently been described in MFS patients without FBN1 gene mutation (2). TGFBR2 gene mutations are associated with LDS and Ehlers-Danlos syndrome which may cause $\operatorname{TAAD}(5,6)$. More than 100 mutations on the TGFBR2 gene have been identified in the $\mathrm{UMD}$, however, the mutation $1,453 \mathrm{G}=>\mathrm{A}$ had not been previously reported. As the father of Case 2 was not available for analysis, it was uncertain whether this mutation was inherited from him or newly acquired. This mutation may not cause familial TAAD because it was not detected in Case 1 and Case 3. To the best of our knowledge, there is no report of FBN1 and TGFBR2 gene mutations found in one patient at the same time. It is unknown how these two mutations affect each other. Recent studies demonstrated that deficiency of fibrillin-1 leads to excessive signaling by TGF- $\beta$, and blockade of TGF- $\beta$ signaling prevents aortic dilatation in a mouse model $(8,9)$. These findings suggest that TGF- $\beta$ signaling may play an important role in type-1 fibrillinopathy. In addition, enhanced TGF- $\beta$ signaling was found in MFS with TGFBR1/2 mutation that is a loss-offunction mutation (6). Therefore, FBN1 and TGFBR2 gene mutations may additively affected the development of TAAD through the rise of the TGF- $\beta$. Indeed, a wide uvula and translucent skin in Case 2 are the features of LDS rather than MFS. Very careful observation is necessary for Case 2 and her child in the future.

\section{References}

1. Robinson PN, Arteaga-Solis E, Baldock C, et al. The molecular genetics of Marfan syndrome and related disorders. J Med Genet 43: 769-787, 2006.
2. Dean JC. Marfan syndrome: clinical diagnosis and management. Eur J Hum Genet 15: 724-733, 2007.

3. Biggin A, Holman K, Brett M, Bennetts B, Ades L. Detection of 
thirty novel FBN1 mutations in patients with Marfan syndrome or a related fibrillinopathy. Hum Mutat 23: 99, 2004.

4. Faivre L, Collod-Beroud G, Loeys BL, et al. Effect of mutation type and location on clinical outcome in 1,013 probands with Marfan syndrome or related phenotypes and FBN1 mutations: an international study. Am J Hum Genet 81: 454-466, 2007.

5. Loeys BL, Schwarze U, Holm T, et al. Aneurysm syndromes caused by mutations in the TGF-beta receptor. N Engl J Med 355: 788-798, 2006.

6. Pannu H, Fadulu VT, Chang J, et al. Mutations in transforming growth factor-beta receptor type II cause familial thoracic aortic aneurysms and dissections. Circulation 112: 513-520, 2005.
7. Sadamatsu K, Tanaka T, Tashiro H, Yasunaga H, Yamamoto K. Intimal flap of ascending aortic dissection with valve-like motion diagnosed by transesophageal echocardiography. Intern Med 44: 1105-1106, 2005.

8. Neptune ER, Frischmeyer PA, Arking DE, et al. Dysregulation of TGF-beta activation contributes to pathogenesis in Marfan syndrome. Nat Genet 33: 407-411, 2003.

9. Habashi JP, Judge DP, Holm TM, et al. Losartan, an AT1 antagonist, prevents aortic aneurysm in a mouse model of Marfan syndrome. Science 312: 117-121, 2006.

(C) 2009 The Japanese Society of Internal Medicine http://www.naika.or.jp/imindex.html 